\title{
The role of inhibition in the production of disfluencies
}

\author{
Paul E. Engelhardt and Martin Corley \\ University of Edinburgh, Edinburgh, Scotland \\ JoEL T. NigG \\ Oregon Health and Science University, Portland, Oregon \\ AND \\ FERNANDA FERREIRA \\ University of Edinburgh, Edinburgh, Scotland
}

\begin{abstract}
Disfluency is a common occurrence in speech and is generally thought to be related to difficulty in the production system. One unexplored issue is the extent to which inhibition is required to prevent incorrect speech plans from being articulated. Therefore, we examined disfluency production in participants with attentiondeficit/hyperactivity disorder (ADHD), which is linked to deficits in inhibitory function and response suppression (Nigg, 2001). Participants completed a sentence production task in which they were presented with two pictures and a verb and their task was to produce a sentence. If inhibition plays a role in preventing incorrect speech plans, we would expect ADHD participants to produce more repetition and repair disfluencies than would non-ADHD controls. The results showed that one subtype of ADHD (i.e., the combined) produced more repair disfluencies as task demands increased. We conclude that the production system relies on inhibitory control in order to prevent errors in language production.
\end{abstract}

Spoken language often contains various types of disfluency. These range from filled pauses, such as $u h$ and um, to corrections (Fox Tree, 1995). Corrections include repetitions and repairs. Repetitions refer to unintended repeats of a word or a string of words (e.g., The ... the other one). Repairs occur when a speaker stops and then starts over with some new word or phrase (e.g., Turn left ... turn right at the light). There has been a great deal of work on the effects of disfluency on language comprehension, and this work has revealed that the comprehension system can be affected by disfluencies in various ways. For example, it is well established from corpus work that disfluencies occur more frequently at the beginning of clauses and other complex constituents (Ford, 1982; Goldman-Eisler, 1968). Bailey and Ferreira (2003) therefore hypothesized that disfluencies may have an impact on the processing of garden path sentences, which involve a choice between a simple and a more complex structure. They found that when a disfluency (i.e., $u h$ ) was placed at the choice point, comprehenders were more likely to pursue the more complex alternative, as compared with a sentence that did not contain a disfluency. This suggests that the comprehension system can use the presence of a disfluency to make decisions about alternate structural analyses (see also Ferreira \& Bailey, 2004).
In another line of work, Arnold, Tanenhaus, Altmann, and Fagnano (2004) investigated the effects of disfluency in a referential communication task. They showed that the presence of a disfluency led to faster identification of an object when it preceded a discourse-new referent, as compared with a referent that had already been established in the discourse. Fox Tree (2001) also found faster recognition for upcoming words in the speech stream when they were preceded by $u h$, as compared with the same utterances that had the disfluency excised. She concluded that disfluency signals a delay and heightens a listener's attention to the upcoming word, which facilitates recognition. Corley, MacGregor, and Donaldson (2007) showed a reduced N400 component for contextually unpredictable words following a disfluency, which suggests that the presence of a disfluency can ease semantic integration (Kutas \& Hillyard, 1980).

Taken together, this body of work demonstrates that disfluencies can have a wide range of beneficial effects on language comprehension (Brennan \& Schober, 2001; Lau \& Ferreira, 2005). It is surprising that there has been comparably less work on the factors that affect the production of disfluency. Work focusing on production has used two main methodologies. The first is the examination of natural language corpora to identify the distribu- 
tion of disfluencies in naturally occurring speech (Clark $\&$ Fox Tree, 2002). The second approach makes use of experimental tasks designed to elicit disfluencies, mainly through manipulations of time pressure and nameability of the objects that a speaker must refer to (Oomen \& Postma, 2001).

One of the main theoretical questions concerning the production of disfluencies is whether they serve as an intentional signal from speaker to listener, or whether they occur as a by-product of difficulty within the language production system. Given the work on the comprehension side, there is little doubt that disfluencies can convey certain types of information, such as the likelihood of a more complex structure or the expectation of a discoursenew referent. Disfluencies have also been argued to convey more high-level (or pragmatic) information, such as a speaker's confidence about what it is that he or she is saying (Brennan \& Williams, 1995; Smith \& Clark, 1993). In addition, corpus work has shown that fillers often occur at conversational boundaries, and so it has also been argued that they serve as signals that a speaker has more to say and wants to "hold" the conversational floor (Clark, 1994).

Clark and Fox Tree (2002) examined two transcribed corpora, and they argued that different types of disfluency perform distinct and highly specific functions. They suggested that speakers use filled pauses (i.e., $u h$ and $u m$ ) as a collateral signal that they are experiencing difficulty. More specifically, they argued that $u h$ is a signal of an upcoming minor delay and that $u m$ is a signal of an upcoming major delay (see also Barr, 2001; Fox Tree, 2001; Fox Tree \& Clark, 1997). Therefore, according to the Clark and Fox Tree account, disfluencies serve as a deliberate and informative signal to a listener.

This account of disfluency production is in contrast to the view that disfluencies occur due to problems in the production system and that listeners are simply taking advantage of regularities in production to help guide lexical and syntactic decisions. Evidence for this view has come from work by O'Connell and Kowal (2005), who examined a corpus of television interviews with then U.S. Senator Hillary Clinton. Crucially, they analyzed the acoustic properties of the filled pauses. O'Connell and Kowal found no evidence to suggest that $u \mathrm{~h}$ and $u m$ signaled an upcoming delay. Therefore, their conclusion was that filled pauses are not reliable cues to the presence of a delay. In another study, Finlayson and Corley (2010) investigated this issue in a study that compared disfluency production in dialogue and in monologue situations, with the hypothesis that if disfluencies are produced with communicative intent, they should occur more often in a dialogue than in a monologue. However, Finlayson and Corley found no difference in the rate of disfluency production when participants were speaking to a confederate, as compared with when they were not (see also Oviatt, 1995). Therefore, the empirical evidence (vs. the transcribed corpus analysis of Clark \& Fox Tree, 2002) suggests that disfluencies are more likely to be due to difficulty in the production system, rather than being an intentional (or collateral) signal of a delay.

\section{The Present Study}

Speech errors and disfluencies have long been used to investigate the cognitive processes that underlie language production (Fromkin, 1971; Garrett, 1975; GoldmanEisler, 1968). The basic production architecture, according to Levelt (1983), is a three-stage model consisting of conceptualization, formulation, and articulation. Many models of language production additionally assume the existence of an internal speech monitor, which is a mechanism whereby speakers check the appropriateness of their speech prior to articulation (Bock \& Levelt, 1994; Dell, 1986; Levelt, 1983, 1989). The monitor is a single centralized mechanism that receives information from the conceptualization and formulation stages. It operates by perceiving internal speech. Phonetic plans, which are generated incrementally (Bock \& Warren, 1985; Christianson $\&$ Ferreira, 2005), are stored in a buffer prior to articulation, and the speech monitor operates on the contents of this buffer. When the monitor detects an error, a self-repair or correction process is initiated, which will lead to either an overt or a covert repair. Therefore, the production system detects errors by listening to internal (prearticulated) speech. A key theoretical issue concerning language production, and one that has remained largely unexplored, is the extent to which the system relies on inhibitory control to suppress unwanted information (Engelhardt, Ferreira, \& Nigg, 2009; Gorfein \& MacLeod, 2007; Meyer, Wheeldon, \& Krott, 2007) and to minimize the production of disfluencies (Blackmer \& Mitton, 1991).

One exception, however, is in the cognitive-aging literature. There have been several studies that investigated disfluency production in older adults, as compared with younger adults (for a review, see Mortensen, Meyer, \& Humphreys, 2006). Moreover, many prominent theories of cognitive decline in aging assume a prominent role for a decrease in inhibitory control (e.g., Hasher \& Zacks, 1988), especially with regard to the regulation of attention and protecting the contents of working memory (Hasher, Zacks, \& May, 1999). However, others argue that the cognitive problems associated with old age are attributable to deficits in both sensory and perceptual processing, which show universal decline with age (Burke \& Osborne, 2007), as well as to a more general cognitive slowing (Salthouse \& Meinz, 1995).

With respect to disfluent speech, older adults have been shown to produce more disfluencies across a range of tasks. These disfluencies are primarily related to lexical retrieval difficulty (Cooper, 1990; Le Dorze \& Bedard, 1998; Sandson, Obler, \& Albert, 1987; SchmitterEdgecombe, Vesneski, \& Jones, 2000), since disfluencies are more common within phrases, rather than between phrases. Consistent with the lack of between-phrase disfluencies, Davidson, Zacks, and Ferreria (2003) found that older and younger adults were equally fluent in a sentence production task that was designed to assess syntactic planning. One area in which age-related inhibition problems have been shown to affect language production is topic maintenance in conversation (Arbuckle, Nohara-LeClair, \& Pushkar, 2000; James, Burke, Austin, \& Hulme, 1998). Older adults, especially in more unconstrained tasks, have 
a tendency to get off topic, and according to an inhibitory deficit account, this is due to older adults' having greater difficulty inhibiting irrelevant information. Therefore, it is not clear that the inhibition problems that affect language production in older adults are necessarily linked to their increased tendency to produce disfluent language. Instead, the tendency to produce more disfluencies seems to be due to slower and more effortful word retrieval.

In the present study, we examined disfluency production in a clinical population suffering from a disorder that has also been linked to deficits in inhibition (Barkley, 1997; Nigg, 2001; Schachar, Tannock, Marriott, \& Logan, 1995). Attention-deficit/hyperactivity disorder (ADHD) is clinically defined by two related but partially distinct symptom domains (American Psychiatric Association, 2000). The inattention domain is characterized by symptoms such as not paying attention, losing things, having difficult staying on task, and so forth. The hyperactivityimpulsivity domain is characterized by symptoms such as fidgeting, running or climbing excessively, blurting out answers, talking excessively, and so forth. Three subtypes of the disorder are possible on the basis of the predominant symptoms. The ADHD-primarily inattentive (PI) subtype reflects elevated symptoms of inattention but not hyperactivity-impulsivity, whereas the ADHDcombined (C) subtype reflects elevated symptoms in both domains. The ADHD-primarily hyperactive-impulsive (PH) subtype is infrequent past childhood, and so, as expected, cases of this subtype were infrequently identified in our sample of adolescents and adults (Hart, Lahey, Loeber, Applegate, \& Frick, 1995; Lahey, Pelham, Loney, Lee, \& Willcutt, 2005; Willcutt, Pennington, \& DeFries, 2000). A diagnostic team evaluated symptoms on the basis of convergence across reporters as detailed in the Method section, and the subtypes were defined on the basis of both current and lifetime symptom status.

The main research question that we addressed in this study was whether the language production process relies on inhibition in order to prevent inappropriate speech plans from being articulated. Following previous work, we assumed that different types of disfluencies would be associated with different processes, and we focused on three types of disfluency: filled pauses, repetitions, and repairs. Previous corpus work has shown that filled pauses tend to occur at sentence-initial positions, which suggests that they are associated with planning. ${ }^{1}$ Repetitions and repairs, in contrast, occur after lexical articulation has been initiated and then some difficulty arises that requires articulation to be suspended. With repetitions, there is a problem, presumably with upcoming words, that prevents a fluent continuation. Clark and Wasow (1998) hypothesized that a speaker repeats some linguistic material in an attempt to restore continuity to the constituent that was interrupted. Repairs occur when the wrong word or phrase has been articulated. In this case, the production system suspends articulation and then starts over with a new word or phrase. For both repetitions and repairs, there is some reformulation that is required before the utterance can continue. If inhibition does play a role in detecting and preventing errors, we would expect ADHD participants to begin speaking without having formulated a plan that affords a fluent utterance or to make outright errors. If this prediction is correct, we should observe a greater number of repetition and repair disfluencies in ADHD participants, as compared with non-ADHD controls. If filled pauses, especially those occurring in sentence-initial positions, are due to planning, we may also observe fewer filled pause disfluencies in the ADHD group (cf. Christenfeld $\&$ Creager, 1996). This result would be the corresponding clue that this group does not plan as much prior to initiating their utterance and, as a result, must make more corrections in midstream.

A secondary goal of the study was to examine several demographic factors on the rate of disfluency production. Bortfeld, Leon, Bloom, Schober, and Brennan (2001) examined corpus data in order to investigate factors that may influence disfluency production, such as the speaker's age, task role (director vs. performer), task difficulty (describing abstract shapes vs. photographs), familiarity of speaker and listener, and gender. They found some distributional differences among the different types of disfluency in relation to task role and difficulty. These results held especially for filled pauses. Filled pauses were more frequent when participants performed the role of director, as compared with performer, and repetition disfluencies were more common when task demands were higherthat is, when participants were required to describe abstract figures, called tangrams, as compared with photographs of children.

Similarly, we were interested in other factors that could influence rates of disfluency production. The first was age. There is evidence to suggest that older adults have more difficulty with word retrieval, as was discussed above (Sandson et al., 1987) and, as a result, produce significantly more disfluencies than do younger and middle-aged adults (Bortfeld et al., 2001; cf. Shewan \& Henderson, 1988). In the present study, we considered the developmental trajectory of disfluency production, focusing more narrowly on adolescents and young adults (from 13 to 35 years of age). We were particularly interested in this age range because of the continued development of cognitive control during this period, which is primarily due to ongoing myelinization of the prefrontal cortex through the late teens and early twenties (Giedd et al., 1999). A second variable that we were interested in was gender. Previous work has shown that males tend to produce more filled pauses and repetitions than do females (Bortfeld et al., 2001; Shriberg, 1996). However, the sexes were found not to differ in the rate at which they produced repairs. Finally, all of the participants in our study completed an assessment of general intelligence (IQ). We believe that this is an important control variable, since people afflicted with ADHD score lower on IQ tests, on average, than do non-ADHD individuals, perhaps secondary to problems with inhibitory control and attention. Therefore, we wanted to be able to control for individual differences in intelligence.

In summary, we examined a large sample of communityrecruited participants. Approximately one third served as non-ADHD controls, and the other two thirds met criteria 
Table 1

Sample Characteristics for the Three Diagnostic Groups

\begin{tabular}{|c|c|c|c|c|c|c|}
\hline \multirow[b]{3}{*}{ Variable } & \multicolumn{6}{|c|}{ Group } \\
\hline & \multicolumn{2}{|c|}{ Controls (86) } & \multicolumn{2}{|c|}{ ADHD-PI (56) } & \multicolumn{2}{|c|}{ ADHD-C (52) } \\
\hline & $M$ & $S D$ & $M$ & $S D$ & $M$ & $S D$ \\
\hline Age (years) & 17.65 & 4.53 & 18.21 & 4.35 & 18.50 & 4.59 \\
\hline Adolescents $(n=129)$ & 15.62 & 1.08 & 15.44 & 1.26 & 15.27 & 1.01 \\
\hline Adults $(n=65)$ & 24.05 & 5.28 & 22.50 & 3.91 & 22.91 & 3.82 \\
\hline \multicolumn{7}{|l|}{ Gender } \\
\hline Male (\%) & 55.2 & & 62.5 & & 82.7 & \\
\hline Female (\%) & 44.8 & & 37.5 & & 17.3 & \\
\hline Education & 12.45 & 2.00 & 12.31 & 1.80 & 12.04 & 1.52 \\
\hline Full-scale IQ & 112.37 & 13.85 & 106.66 & 14.19 & 105.66 & 12.44 \\
\hline Conner's (1) & 48.27 & 7.21 & 65.94 & 10.22 & 67.78 & 8.19 \\
\hline Conner's (2) & 47.99 & 7.31 & 57.83 & 11.24 & 72.57 & 10.08 \\
\hline
\end{tabular}

Note-Conner's scores are $t$ scores; (1) is "cognitive problems," which is closely related to $D S M-I V$ inattentive symptoms, and (2) is hyperactive-impulsive symptoms. Values represent the average of informant and self-report for adults and the average of mother and teacher ratings for adolescents. ADHD-PI, attention-deficit/hyperactivity disorderprimarily inattentive; ADHD-C, ADHD-combined.

for ADHD. As was mentioned previously, the ADHD$\mathrm{PH}$ subtype is rare to nonexistent past childhood. Therefore, our ADHD sample consisted of approximately half ADHD-PI and half ADHD-C participants. Since this is the first study to investigate disfluency production with this disorder and because the symptom domains partially overlap, it is difficult to make specific predictions regarding the different subtypes. However, considering that the ADHD-C subtype is generally more severe and involves impulsive language behavior (i.e., talking excessively, blurting out answers in class, and/or not awaiting turns in conversation), we might expect this subtype to show the clearest difficulty managing language production. The primary goal of this study was to investigate whether the tendency to produce certain types of disfluency is related to inhibitory control. More specifically, we expected ADHD participants to produce more repetition and repair disfluencies, as compared with the non-ADHD group. Conversely, we expected the non-ADHD group to produce more filled pauses, since these are hypothesized to be related to planning. The secondary goal of the study was to examine the effect of several demographic factors on the overall rates of disfluency production.

\section{METHOD}

\section{Participants}

One hundred ninety-four participants (13-35 years old) who were recruited for a large-scale ADHD study participated. The participants were community recruited, which resulted in as broad and representative a sample as possible. Table 1 shows the demographic data for the three diagnostic groups: controls, ADHD-PI, and ADHD-C. Table 1 shows that the groups differed as expected with regard to the typical clinical profile of ADHD. The participants in this study completed a semistructured clinical interview and an assessment of IQ (Wechsler, 1997a, 1997b, 2001). IQ was estimated using a reliable and valid five-subtest short form of the WAIS-III (16 years and younger) and WISC-IV (17 years and older) (Sattler, 2001). The subtests were picture completion, vocabulary, similarities, arithmetic, and matrix reasoning. ${ }^{2}$

For adolescents, an interview of the parents was administered to ascertain current and lifetime symptoms of ADHD and all Axis I dis- orders in the same manner. Teacher ratings were obtained to evaluate cross-situational symptom display. For adults, current ADHD symptoms were ascertained by self-report and by interview with a second informant - typically, a spouse, roommate, or close friend. The parent, teacher, and informant interviews were conducted by clinically trained interviewers via telephone after appropriate consent procedures.

For all the participants, a diagnostic team consisting of a licensed clinical psychologist and a board-certified psychiatrist arrived at a best-estimate diagnosis (Faraone et al., 2000). The same team evaluated all the cases. Each member independently reviewed all available information from all interviews (including staff notes and observations) to arrive at a clinical judgment about ADHD present or absent, ADHD subtype, and any comorbid disorders. Clinical interviewers rated and noted evidence of impairment (i.e., a rating of at least "moderate" on the KSAD rating scale; Puig-Antich \& Ryan, 1986), and the diagnostic team required such evidence to make a diagnosis. Whereas the $D S M-I V$ does not provide adult-specific criteria, the procedure here was conservative in requiring the adults to meet the $D S M-I V$ criteria developed for younger ages. This procedure minimizes false positives because those assigned to the ADHD group had ample evidence of the disorder for their age. On the other hand, false negatives (i.e., ADHD cases in the control group) were minimized by requiring four or fewer symptoms of inattention and hyperactivity, no past history of ADHD diagnosis (by both self-report and informant report), and rating scale data in the normal range, as compared with national norms.

Interclinician agreement on the presence or absence of ADHD was satisfactory $(k=.80)$, and agreement on ADHD subtype was also adequate, ranging from $k=.74$ to $k=.90$. Discrepancies were handled by conference of the clinicians, and it happened that consensus was readily achieved in all cases. If consensus could not have been achieved, that case would have been excluded. All the participants were tested after suitable periods of medication washout. Additional details of the evaluation and diagnosis procedures can be obtained in Carr, Nigg, and Henderson (2006) and Martel, Nikolas, and Nigg (2007).

\section{Sentence Production Task}

In language production, the order in which words or concepts are activated tends to influence the order of words in a subsequent sentence (Bock, 1987; Bock \& Warren, 1985; Ferreira \& Engelhardt, 2006). In the present study, we took advantage of this incrementality in the production system to systematically vary the level of difficulty in a sentence production task. On each trial, the participants were presented with one animate object and one inanimate object, along 


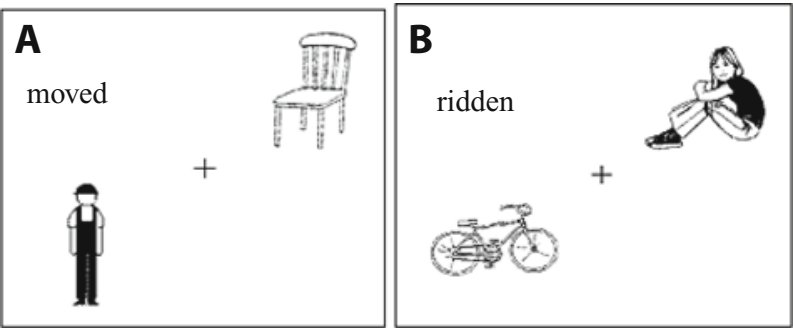

Figure 1. Example stimuli. (A) An ambiguous verb. (B) A participle verb.

with a printed verb that was either an unambiguous participle verb (e.g., ridden) or a verb that was ambiguous between past tense and past participle forms (e.g., dropped). On half of the trials, the inanimate object was presented first, which biases toward a passive structure (see Figure 1). On the other half of the trials, the animate object was presented first, and this ordering favors an active structure. The participle verbs bias toward passives. ${ }^{3}$ In both spoken and written English, there is a strong general preference for actives over passives (Dick \& Elman, 2001), and we expected more disfluencies when the verb was a participle and when the object order and verb bias conflicted. The conflicting conditions were cases in which the animatefirst object order (active bias) was paired with a participle verb (passive bias) and those in which the inanimate-first object order (passive bias) was paired with an ambiguous verb (active bias).

For this task, the objects appeared one after the other and were followed by the verb. Each trial began with a fixation cross presented at the center of the computer screen. This was the participants' cue that they could press the space bar to see the first object. After $1 \mathrm{sec}$, a second object appeared. It was followed $1 \mathrm{sec}$ later by the verb. The participants were instructed to begin speaking as soon as possible. The participants were given 4 practice trials with feedback, followed by 36 regular session trials. The stimulus materials consisted of 72 line drawings of easily nameable objects and 36 verbs. Half of the drawings were of animate objects, and half were of inanimate objects. Eighteen of the verbs were ambiguous, meaning that the past tense and past participle forms were identical (e.g., moved), and 18 were unambiguous participle verbs (e.g., ridden). Participle verbs included both irregulars (e.g., torn) and -en affixes.

\section{Design and Procedure}

The design was $3 \times 2 \times 2$ (diagnostic group $\times$ object order $\times$ verb type). Object order and verb type were within subjects, and diagnostic group was between subjects. Object order indicates which drawing, animate or inanimate, was presented first. Verb type was either ambiguous or a participle. Participle verbs are biased toward passives and are, in general, more difficult, because they have fewer syntactic options, as compared with ambiguous verbs (see note 3 ).

Participant responses were recorded to audiotape and then transcribed and coded. Three types of disfluencies were examined: filled pauses (i.e., $u h, u m$, and $e r$ ), repetitions, and repairs (Ferreira, Lau,
\& Bailey, 2004). The data were transcribed and coded by two trained research assistants who transcribed and coded the same data from 50 participants. Interrater reliability was excellent; there was $95.2 \%$ agreement. The few disagreements that did exist were reevaluated and resolved. Each research assistant then coded approximately half of the remaining data. The order of trials was randomly determined for each participant, and the entire experimental session lasted approximately $30 \mathrm{~min}$.

\section{RESULTS}

For all of the following analyses, the data were screened for outliers, and the proportions were transformed to rationalized arcsine units (Studebaker, 1985). Individual participant means that differed from the condition mean by more than five standard deviations were replaced with the mean for that group in that condition. (This affected only eight data points: six filled pauses and two repetitions.) The total number of sentences analyzed was $6,927 .{ }^{4}$ The total number of words produced was approximately 43,023. There were 691 disfluencies (218 filled pauses, 132 repetitions, 341 repairs). Table 2 shows the correlations between the demographic variables and the dependent variables.

We began the analysis by collapsing across the four within-subjects conditions. The proportion of disfluencies produced per sentence is broken down by the three diagnostic groups in Figure 2. The results of three oneway ANOVAs showed only a significant effect for repair disfluencies $[F(2,193)=4.72, p<.01]$. Paired comparisons showed that the ADHD-C group produced more repairs than did both the ADHD-PI group $[t(106)=-2.65$, $p<.01]$ and the controls $[t(136)=-2.87, p<.01]$. These results show that there was no difference between groups in the rate of filled pauses and repetitions. However, the ADHD-C group was significantly more likely to produce a repair disfluency, as compared with the other two groups. Next, we turn to performance differences in the four within-subjects conditions, in order to examine whether the groups differed as task demands increased.

\section{Filled Pauses}

The three-way mixed model ANOVA conducted on the proportion of utterances containing a filled pause disfluency showed only a main effect of verb type (see Figure 3). The participle verbs yielded more filled pauses than did the ambiguous verbs $[F(1,190)=11.64, p<.001]$. This was expected because the participle verbs had fewer syn-

Table 2

Correlations Between Demographic Variables and Dependent Variables

\begin{tabular}{lccccccc}
\hline \multicolumn{1}{c}{ Variable } & 1 & 2 & 3 & 4 & 5 & 6 & 7 \\
\hline 1. Age & - & -.09 & $.16^{*}$ & $.83^{* *}$ & .01 & $-.15^{*}$ & -.09 \\
2. Gender & & - & -.02 & -.04 & .06 & -.12 & -.05 \\
3. IQ & & & - & $.21^{* *}$ & -.01 & $-.16^{*}$ & $-.19^{*}$ \\
4. Education & & & & - & .04 & $-.19^{*}$ & -.12 \\
5. Filled pauses & & & & & - & .10 & .13 \\
6. Repetitions & & & & & - & .09 \\
7. Repairs & & & & & & - \\
\hline Note- $N=194$. & ${ }^{*} p<.05$. & ${ }^{* *} p<.01$. & & & &
\end{tabular}




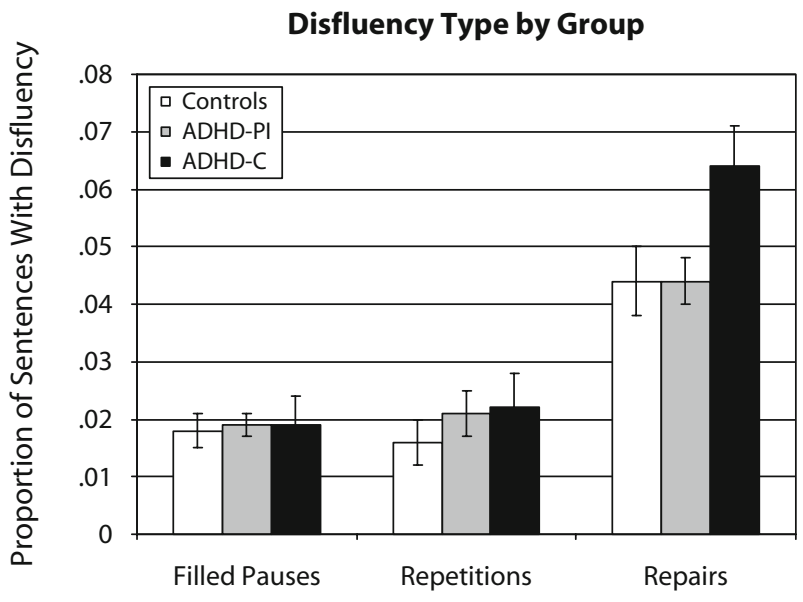

Disfluency Type

Figure 2. Proportion of disfluencies produced by each group. Error bars show the standard errors of the means. ADHD-PI, attention-deficit/hyperactivity disorder-primarily inattentive; ADHD-C,ADHD-combined.

tactic options and, as a result, were more difficult to incorporate into a sentence than were the ambiguous verbs. The higher rate of filler production in these two conditions was likely due to increased planning difficulty; however, there was no effect of diagnostic group.

\section{Repetitions}

The three-way mixed model ANOVA conducted on the proportion of utterances containing a repetition showed that two main effects were significant. As in the previous analysis, there were more repetitions with participle verbs than with ambiguous verbs $[F(1,190)=9.40, p<.01$; see Figure 4]. The main effect of object order showed more repetitions when the inanimate object was presented first $[F(1,190)=4.54, p<.05]$. Both of these effects are consistent with the general predictions that participle verbs are more difficult than ambiguous verbs and that people have a general preference for actives over passives. The latter finding suggests that although the inanimate-first object order should prime a passive structure, due to incrementality, the general preference for actives still leads to a processing cost for the less preferred passive form.

\section{Repairs}

The three-way mixed model ANOVA conducted on the proportion of utterances with a repair showed that all three main effects were significant. As in the previous analyses, there were more repairs with participle verbs than with ambiguous verbs $[F(1,190)=131.93, p<.001]$. The main effect of object order showed more repairs when the inanimate object was presented first than when the animate object was presented first $[F(1,190)=5.39, p<.05]$. Finally, there was a main effect of group $[F(2,190)=4.21$, $p=.01]$, and this result was primarily due to the ADHD-C group. Follow-up one-way ANOVAs conducted on each of the four within-subjects conditions showed significant differences in the inanimate-first/ambiguous-verb condition
$[F(2,190)=4.20, p<.05]$ and marginal differences in the animate-first/participle-verb condition $[F(2,190)=2.52$, $p=.08$; see Figure 5]. In the inanimate-first/ambiguousverb condition, the ADHD-C group was significantly more likely to produce a repaired utterance, as compared with both of the other groups, ADHD-PI $[t(105)=2.70$, $p<.01]$ and controls $[t(135)=2.06, p<.05]$. For the animate-first/participle-verb condition, the ADHD-C group was marginally worse than the ADHD-PI group $[t(105)=1.82, p=.07]$ and was significantly worse than controls $[t(135)=2.09, p<.05]$.

There was also an interaction between verb type and object order $[F(1,190)=7.69, p<.01]$, and the interaction, in this case, was driven by differences with the participle verbs. The inanimate-first order resulted in more repairs, as compared with the animate-first order $[t(192)=-3.14, p<.01]$. In contrast, there was no difference based on object order with the ambiguous verbs $(p>.05)$.

To summarize, we observed group differences only for repair disfluencies. These group differences were in the two conditions in which the verb bias and the animacy order conflicted. Recall that participle verbs bias toward passive structures and are more difficult than ambiguous verbs. Therefore, the most difficult condition was one in which the participle verb was paired with the animatefirst object order. The results showed that the ADHD-C group produced a marginally greater number of repairs in this condition and that they were significantly worse with the ambiguous verbs when the inanimate picture was presented first. This is the other condition in which the verb bias and the object order conflict, because the ambiguous verbs favor active structures and the inanimate-first order biases toward a passive structure. On the basis of these data, we conclude that deficits in inhibitory control do influence the rate of repair disfluencies. In the Discussion section, we will discuss possible reasons why the two ADHD subtypes showed a different pattern of results.

Recall that the secondary goal of this study was to rule out other possible cognitive control explanations of group differences, mainly related to IQ. Examining the correlations in Table 2 reveals that IQ was significantly (negatively) correlated with repairs. Therefore, we wanted to determine whether IQ mediated the increased likelihood of a repair disfluency in the ADHD-C group, especially because this group had significantly lower IQ scores $(p<$ .01 ), as compared with the non-ADHD group (see Table 1). To examine whether IQ mediated the relationship between the ADHD-C group and the rate of repair disfluencies, we conducted mediation tests according to the procedures recommended by Barron and Kenny (1986). We started with the number of repairs in the inanimate-first/ambiguousverb condition, and for these analyses, we compared the ADHD-C group with controls. The results from a simple regression using group as a predictor of the proportion of repairs showed a significant effect $[F(1,135)=4.22, p<$ $\left..05, R^{2}=.03\right]$. On this basis, we can conclude that diagnostic group (controls vs. ADHD-C) predicts the proportion of repair disfluencies (see Path $\mathrm{C}$ in Figure 6). We next tested Path A to determine whether diagnostic group pre- 


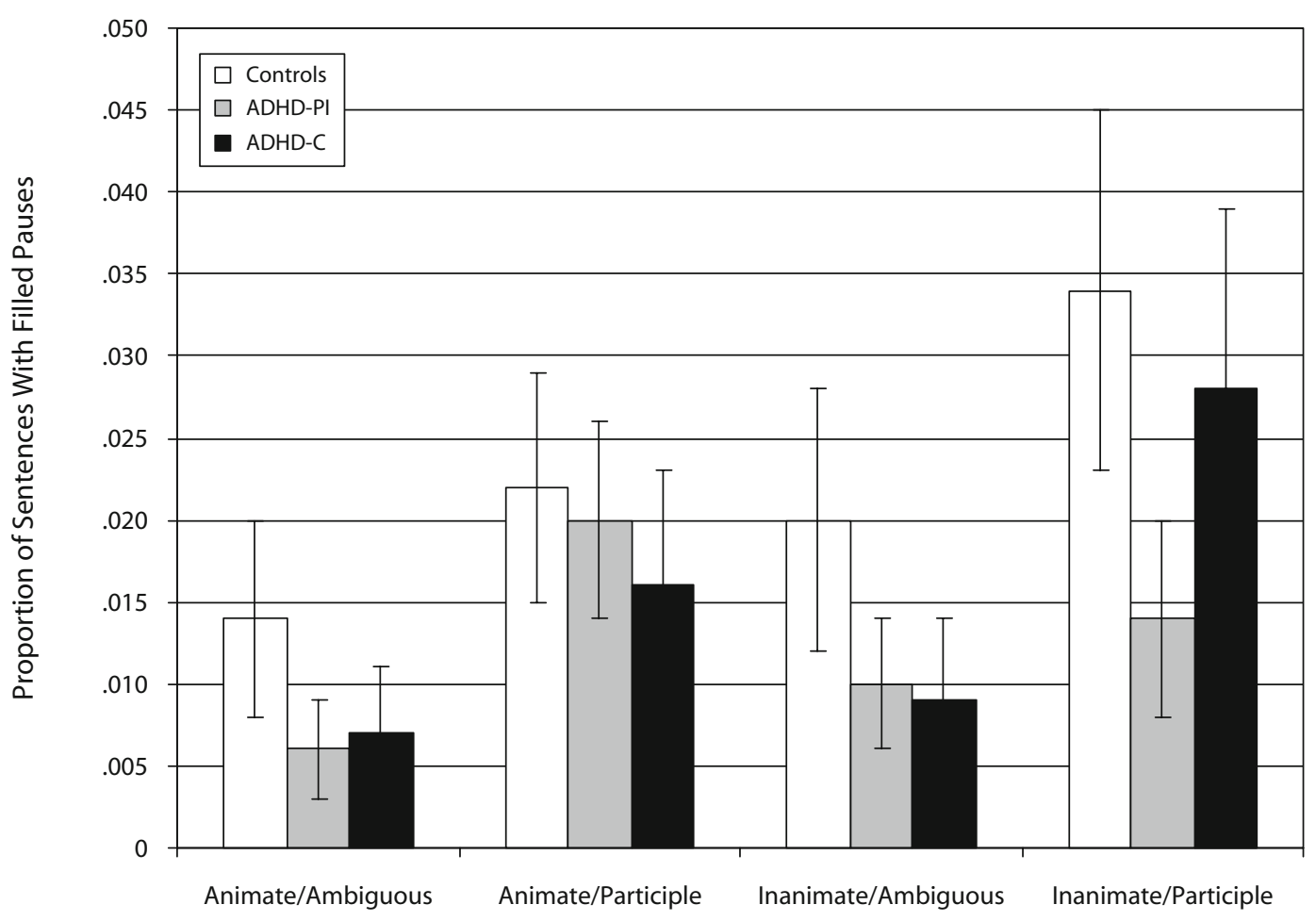

Condition

Figure 3. Proportion of filled pauses produced in each of the conditions by the three diagnostic groups. Error bars show the standard errors of the means. ADHD-PI, attention-deficit/hyperactivity disorder-primarily inattentive; ADHD-C, ADHD-combined.

dicts IQ. The results from a simple regression showed that group was a significant predictor of IQ $[F(1,135)=8.16$, $\left.p<.01, R^{2}=.057\right]$. The final step in the mediation test was to include both variables as independent variables and proportion of repairs as the dependent variable. If Path B was significant and Path $\mathrm{C}$ was nonsignificant, this would suggest that the effect was completely mediated. However, the results from the regression model testing both IQ and diagnostic group as predictors showed that IQ was not significant $(p>.08)$ but diagnostic group was significant $[t(132)=2.16, p<.05, \beta=.191]$. Because Path B was not significant and Path $C$ remained significant with IQ included in the model, we can conclude that IQ did not mediate the group differences in this condition.

We also examined whether IQ mediated performance in the animate-first/participle-verb condition. The results of a simple regression testing group (i.e., controls vs. ADHD-C) as a predictor of the proportion of repairs $($ Path C) showed a significant effect $[F(1,135)=4.36$, $\left.p<.05, R^{2}=.031\right]$. The model testing Path A in Figure 6 showed that group was a significant predictor of IQ $\left[F(1,135)=8.16, p<.01, R^{2}=.057\right]$. However, when both variables were included and regressed onto the number of repair disfluencies, the results showed, as in the previous analysis, that IQ was not a significant predic- tor $(p>.65)$. In this model, diagnostic group was only a marginal predictor $[t(132)=1.91, p=.08, \beta=.169]$. On the basis of both sets of mediation tests, we conclude that the group differences we observed are unlikely to have been due to underlying differences in IQ. Rather, it is more likely that the increased likelihood of the ADHD-C group's producing a repair disfluency was caused by problems associated with inhibitory control and/or response suppression failures.

A further goal of this study was to examine several demographic variables with respect to the production of disfluencies. There were no variables that correlated with filled pause disfluencies (see Table 2). However, we did find that age, IQ, and years of education were negatively correlated with repetition disfluencies. Age and number of years of education are, of course, highly correlated. ${ }^{5}$ We therefore used age and IQ score as predictors of the proportion of repetitions averaged across the four withinsubjects conditions. We did not include diagnostic group, because there were no significant group differences for repetitions. The model testing age and IQ as predictors of repetitions was significant $[F(2,186)=4.76, p<.01$, $\left.R^{2}=.049\right]$. In addition, the results from this model showed that age was a significant predictor $[t(186)=-2.20, p<$ $.05 ; \beta=-.159]$ but that IQ was only a marginally signifi- 
Proportion of Repetitions

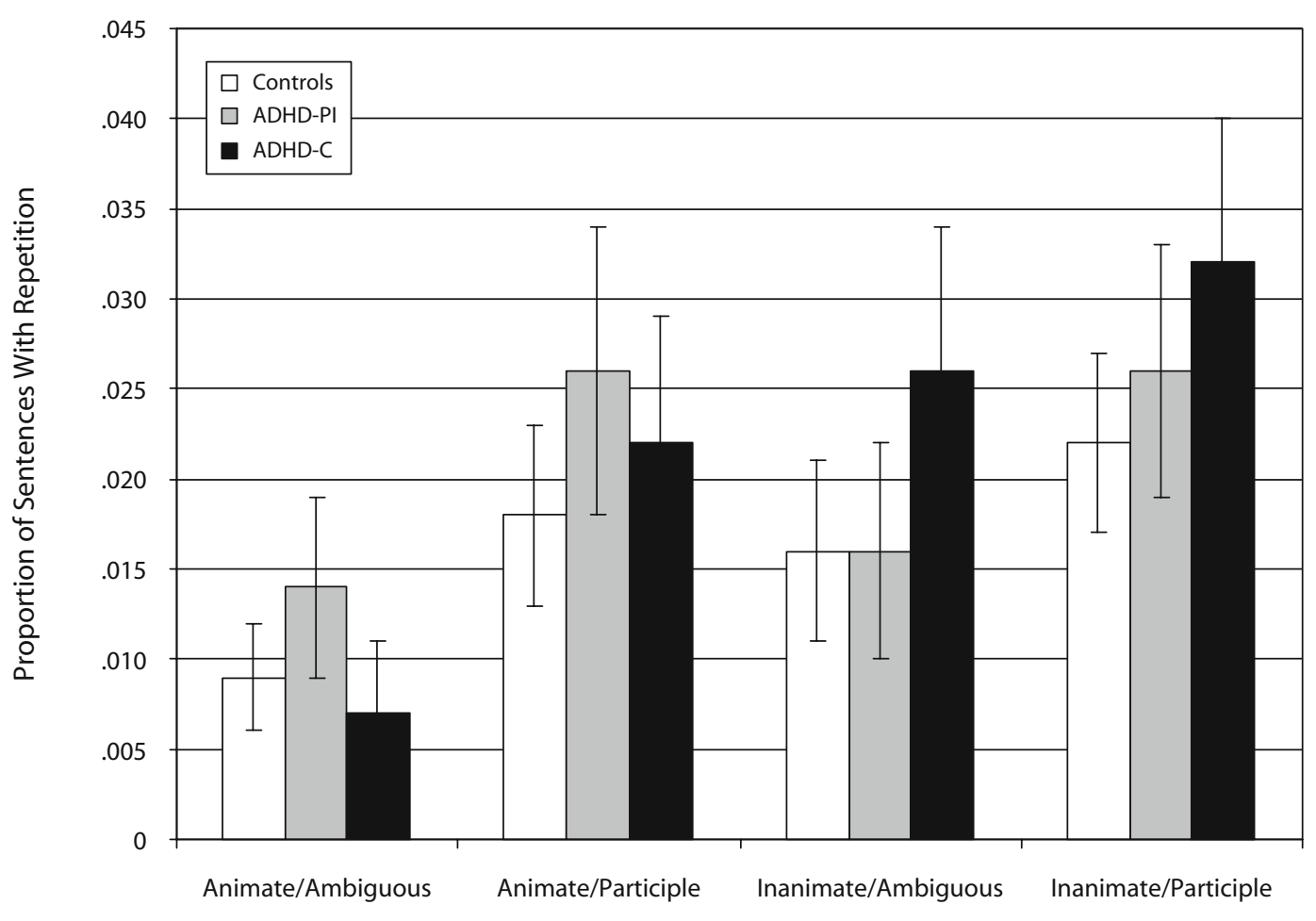

Condition

Figure 4. Proportion of repetitions produced in each of the conditions by the three diagnostic groups. Error bars show the standard errors of the means. ADHD-PI, attention-deficit/hyperactivity disorder-primarily inattentive; ADHD-C,ADHD-combined.

cant predictor $[t(186)=-1.80, p=.074 ; \beta=-.130]$. These results indicate that as people get older, they produce repetition disfluencies less often. A similar but not significant pattern occurs for participants with higher IQ. Gender did not have an effect on any of the three types of disfluencies that we examined.

\section{DISCUSSION}

The results from this study showed more disfluencies when the participants were presented with participle verbs. This effect held for filled pauses, repetitions, and repairs. There was a significant effect of object order on both repetitions and repairs. In both cases, there were more disfluencies when the inanimate object was presented before the animate object. There was also a significant interaction between verb type and object order in the number of repairs. That interaction was such that there was a significant difference between the two object orders with the participle verbs but no difference based on object order with the ambiguous verbs.

The primary goal of this study was to investigate the role of inhibition in the production of disfluencies. The main theoretical models of ADHD posit that primary deficits in inhibition and response suppression lead to secondary (or downstream) problems, such as poor attention, distracta- bility, and impulsive behavior (Barkley, 1997; Nigg, 2001, 2006). It is also known that children with ADHD have problems with pragmatic aspects of language output, such as talking excessively, interrupting others, and coordinating the taking of turns in conversation (Lorch et al., 2000; Purvis \& Tannock, 1997; Rashid, Morris, \& Morris, 2001; Redmond, 2004). In this study, the main hypothesis that we were interested in was whether ADHD participants would be more likely to produce corrections (i.e., repetitions and repairs) and less likely to produce filled pauses, as compared with the non-ADHD control group.

Models of language production assume that the system relies on an internal speech monitor, which detects and filters out errors prior to articulation. If the system relies on inhibition or "mental brakes," so to speak, we would expect the ADHD group to have a reduced ability to detect and prevent inappropriate speech plans from being articulated. Filled pause disfluencies, in contrast, have been linked to planning, and similar to what has been reported previously, over three quarters of the filled pauses $(76 \%)$ in our study occurred at the beginning of the sentence. We hypothesized that the ADHD group might also be less likely to produce filled pauses, as compared with the control group. However, the results showed no difference between diagnostic groups in the number of filled pauses. Our results did show that the ADHD-C subtype 


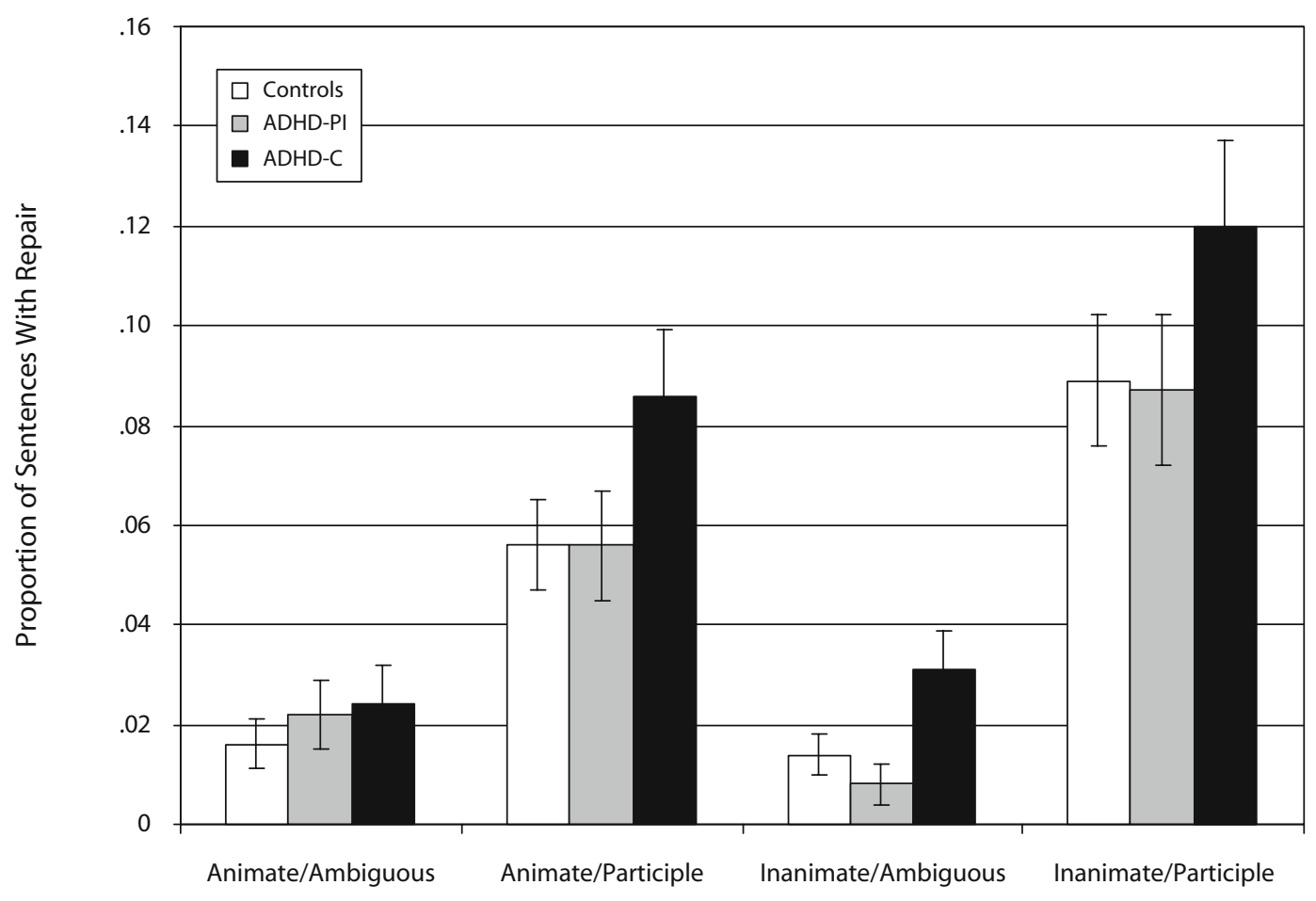

Condition

Figure 5. Proportion of repairs produced in each of the conditions by the three diagnostic groups. Error bars show the standard errors of the means. ADHD-PI, attention-deficit/hyperactivity disorder-primarily inattentive; ADHD-C,ADHD-combined.

was more likely to produce repair disfluencies than were both the ADHD-PI group and controls. These differences were observed in the two most difficult conditions - that is, when the object order and the verb biases conflicted with one another. This result suggests that inhibition does play a role in language production and that it is related to the tendency to have to change words midsentence.

We did not observe significant group differences for either filled pauses or repetitions, which is interesting because it suggests that these types of disfluency are different from repairs. We hypothesized that the repetitions and repairs would pattern similarly and that both would be different from filled pauses. Repairs occur when the wrong word has been selected and the production system does not detect the error prior to articulation. Repetitions, in contrast, are thought to be related to a problem with a word or phrase that occurs later in the sentence from the point where the repetition happened. The problem, in this case, could be that the lack of an adequate plan prevents continuation, or it could be that a wrong word has been selected and the system is in the process of correcting the error and, as a result, must suspend articulation. Clark and Wasow (1998) hypothesized that the repeated linguistic material occurs because it is easier for the speaker to start over at the beginning of the constituent, rather than continuing at the point of suspension. It also serves to mini- mize the disruption. Our results cannot determine what problem in the system caused repetitions to occur. However, the fact that the repetitions patterned similarly to filled pauses does lead us to speculate that they are more likely to be related to difficulties in planning speech.

Another important finding that emerges from this study is that the two ADHD subtypes dissociated from one another. We believe that this pattern can be explained by considering the symptom domains that define the subtypes. The ADHD-PI group shows high levels of symptoms in the inattentive domain. The combined subtype, in contrast, shows high levels of symptoms in both

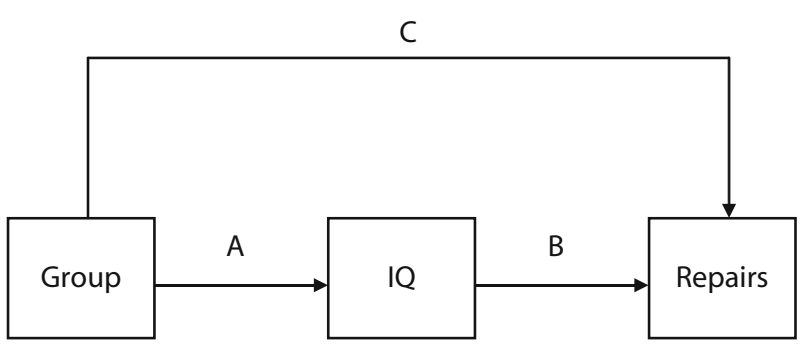

Figure 6. Path diagram showing possible mediation effect of IQ on the relationship between diagnostic group and the proportion of repair disfluencies. 
the inattentive symptom domain and the hyperactiveimpulsive symptom domain. The latter subtype has been more closely linked to (behavioral and motor) response control problems. Therefore, it is not at all surprising that the ADHD-C group produced more repairs, because language production involves both response suppression and a behavioral-output/motor-control component.

The secondary goal of the study was to examine the effects of several demographic and cognitive control variables on the rates of disfluency production. The main cognitive control factor that we looked at was IQ, and we found that variance in IQ was negatively correlated with both repetitions and repairs. In the results, we focused on the relationship between IQ and repairs primarily because this was the type of disfluency that showed an effect of diagnostic group. The results from mediation analyses indicated that differences in IQ did not mediate the increased likelihood that the ADHD-C group would produce a repair disfluency, as compared with controls. Nonetheless, it is interesting to have observed a relationship between IQ and production of disfluencies, since it suggests that some problems associated with language production may be linked to general intelligence, and not to specific components of the language production system. At this time, we do not have specific hypotheses concerning the link between general intelligence and disfluent speech. However, we would point out that verbal ability measures (e.g., phonetic coding, lexical knowledge, grammatical sensitivity, word fluency) typically have some of the highest factor loadings ( . .45-.50) on general intelligence (Carroll, 1993; Deary, 2001). Additional work is required to determine whether the tendency to be disfluent is similar to the other types of verbal ability measures, which are assumed to be a form of crystallized intelligence, or whether fluency in language production is an aspect of fluid intelligence (Engle, Kane, \& Tuholski, 1999).

Finally, we examined the effects of two demographic variables on the rates of disfluency production. Age was a significant predictor of the number of repetitions, which suggests that, over the course of development from adolescence to adulthood, there is a decreasing likelihood of repeating words midsentence. This pattern is different from the findings of Bortfeld et al. (2001). They found a significant linear trend in which people made progressively more disfluencies over time. However, Bortfeld et al.'s sample ranged from 24 to 72 years of age. If we combine those results with ours, it suggests that disfluency production may follow a U-shaped distribution with the bottom occurring in the late 20 s to early 30 s. We did not observe any differences in the rate of disfluencies based on gender, which is interesting for two reasons. The first is that there is some evidence to suggest that females with ADHD have more pronounced language problems (Berry, Shaywitz, \& Berry, 1985). The second is that two previous studies, Bortfeld et al. (2001) and Shriberg (1996), reported that men produced more disfluencies than did women. In both studies, it was observed that men produced more filled pauses, and Bortfeld et al. also reported that men produced more repetitions. Our results indicated that males produced only slightly more repetitions than did females $(p=.105)$ but that they produced essentially the same number of filled pauses and repairs.

\section{Conclusions}

As was expected, disfluency rates increased when speakers were faced with more difficult verbs and when the order of the depicted objects conflicted with the preferred sentence structure. On the basis of the results from this study, we draw several conclusions. The first is that there is an aspect of preventing repair disfluencies in the language production system that is related to inhibitory control. Crucially, this effect is not mediated by differences in IQ. Second, the finding that repairs are distributed somewhat differently from filled pauses and repetitions supports the idea that they arise from different processes. Pauses and repetitions appear to occur when the production system requires more time to plan, whereas repairs are linked to inadequate planning's requiring speakers to backtrack and correct their utterances in midstream. The third conclusion concerns development, and our results indicate that, at least for repetitions, there is a negative relationship between disfluency production and age, from late adolescence to young adulthood. On the basis of the present and previous work, we speculate that rates of repetition production may follow a U-shaped distribution. Overall, this study suggests that the language production system relies on inhibitory control in order to prevent inappropriate words and word sequences from being articulated.

\section{AUTHOR NOTE}

This research was supported by National Institutes of Health, National Institute of Mental Health Grant R01-MH63146 to J.T.N. and F.F. The authors thank Laurie A. Carr and Elizabeth Davis for their help in collecting the data. Correspondence concerning this article should be addressed to P. E. Engelhardt, Department of Psychology, University of Edinburgh, 7 George Square, Edinburgh EH8 9JZ, Scotland (e-mail: paul.engelhardt@ed.ac.uk).

\section{REFERENCES}

American Psychiatric Association (2000). Diagnostic and statistical manual of mental disorders (4th ed., revised). Washington, DC: Author.

Arbuckle, T. Y., Nohara-LeClair, M., \& Pushkar, D. (2000). Effect of off-target verbosity on communication efficiency in a referential communication task. Psychology \& Aging, 15, 65-77.

Arnold, J. E., Tanenhaus, M. K., Altmann, R. J., \& Fagnano, M. (2004). The old and thee, uh, new: Disfluency and reference resolution. Psychological Science, 15, 578-582.

Bailey, K. G. D., \& Ferreira, F. (2003). Disfluencies affect the parsing of garden-path sentences. Journal of Memory \& Language, 49, 183-200.

BARKLEY, R. A. (1997). Behavioral inhibition, sustained attention, and executive functions: Constructing a unifying theory of ADHD. Psychological Bulletin, 1, 65-94.

BARR, D. J. (2001). Trouble in mind: Paralinguistic indices of effort and uncertainty in communication. In C. Cavé, I. Guaïtella, \& S. Santi (Eds.), Oralité et gestualité: Interactions et comportements multimodaux dans la communication (pp. 597-600). Paris: L'Harmattan.

BARRON, R. M., \& KENNY, D. A. (1986). The moderator-mediator variable distinction in social psychological research: Conceptual, strategic, and statistical considerations. Journal of Personality \& Social Psychology, 51, 1173-1182.

Berry, C. A., Shaywitz, S. E., \& Berry, B. A. (1985). Girls with attention deficit disorder: A silent minority? A report on behavior and cognitive characteristics. Pediatrics, 76, 801-809. 
Blackmer, E. R., \& Mitton, J. L. (1991). Theories of monitoring and timing of repairs in spontaneous speech. Cognition, 39, 173-194.

Bock, J. K. (1987). An effect of the accessibility of word forms on sentence structures. Journal of Memory \& Language, 26, 119-137.

Bock, J. K., \& Levelt, W. J. M. (1994). Language production: Grammatical encoding. In M. A. Gernsbacher (Ed.), Handbook of psycholinguistics (pp. 945-984). San Diego: Academic Press.

Bock, J. K., \& WARren, R. K. (1985). Conceptual accessibility and syntactic structure in sentence formulation. Cognition, 21, 47-67.

Bortfeld, H., Leon, S. D., Bloom, J. E., Schober, M. F., \& BrenNAN, S. E. (2001). Disfluency rates in spontaneous speech: Effects of age, relationship, topic, role, and gender. Language \& Speech, 44, 123-147.

Brennan, S. E., \& Schober, M. F. (2001). How listeners compensate for disfluencies in spontaneous speech. Journal of Memory \& Language, 44, 274-296.

BrennAN, S. E., \& WiLliams, M. (1995). The feeling of another's knowing: Prosody and filled pauses as cues to listeners about the metacognitive states of speakers. Journal of Memory \& Language, 34, 383398.

Burke, D. M., \& Osborne, G. (2007). Aging and inhibition deficits: Where are the effects? In D. S. Gorfein \& C. M. MacLeod (Eds.), Inhibition in cognition (pp. 163-183). Washington, DC: American Psychological Association.

CArr, L. A., Nigg, J. T., \& Henderson, J. M. (2006). Attentional versus motor inhibition in adults with attention deficit hyperactivity disorder. Neuropsychology, 20, 430-441.

Carroll, J. B. (1993). Human cognitive abilities: A survey of factoranalytic studies. New York: Cambridge University Press.

Christenfeld, N., \& Creager, B. (1996). Anxiety, alcohol, aphasia, and ums. Journal of Personality \& Social Psychology, 70, 451-460.

Christianson, K., \& Ferreira, F. (2005). Conceptual accessibility and sentence production in a free word order language (Odawa). Cognition, 98, 105-135.

Clark, H. H. (1994). Managing problems in speaking. Speech Communication, 15, 243-250.

Clark, H. H., \& Fox Tree, J. E. (2002). Using $u$ h and um in spontaneous speaking. Cognition, 84, 73-111.

Clark, H. H., \& Wasow, T. (1998). Repeating words in spontaneous speech. Cognitive Psychology, 37, 201-242.

Cooper, P. V. (1990). Discourse production and normal aging: Performance on oral picture description tasks. Journal of Gerontology: Psychological Sciences, 45, 210-214.

Corley, M., MacGregor, L. J., \& Donaldson, D. I. (2007). It's the way that you, er, say it: Hesitations in speech affect language comprehension. Cognition, 105, 658-668.

Davidson, D. J., Zacks, R. T., \& Ferreira, F. (2003). Age preservation of the syntactic processor in production. Journal of Psycholinguistic Research, 32, 541-566.

DEARY, I. J. (2001). Intelligence: A very short introduction. Oxford: Oxford University Press.

DeLL, G. S. (1986). A spreading-activation theory of retrieval in sentence production. Psychological Review, 93, 283-321.

Dick, F., \& Elman, J. L. (2001). The frequency of major sentence types over discourse levels: A corpus analysis. Center for Research in Language Newsletter, 13, 3-18.

Engelhardt, P. E., Ferreira, F., \& Nigg, J. T. (2009). Priming sentence production in adolescents and adults with attention-deficit/ hyperactivity disorder. Journal of Abnormal Child Psychology, 37, 916-928

Engelhardt, P. E., Nigg, J. T., Carr, L. A., \& Ferreira, F. (2008). Cognitive inhibition and working memory in attention-deficit/hyperactivity disorder. Journal of Abnormal Psychology, 117, 591-605.

Engle, R. W., Kane, M. J., \& TuHOLSKI, S. W. (1999). Individual differences in working memory capacity and what they tell us about controlled attention, general fluid intelligence and functions of the prefrontal cortex. In A. Miyake \& P. Shah (Eds.), Models of working memory: Mechanisms of active maintenance and executive control (pp. 102-134). New York: Cambridge University Press.

Faraone, S. V., Biederman, J., Spencer, T., Wilens, T., Seidman, L. J., Mick, E., \& Doyle, A. E. (2000). Attention-deficit/hyperactivity disorder in adults: An overview. Biological Psychiatry, 48, 9-20.
Ferreira, F., \& Bailey, K. G. D. (2004). Disfluencies and human language comprehension. Trends in Cognitive Sciences, 8, 231-237.

Ferreira, F., \& Engelhardt, P. E. (2006). Syntax and production. In M. Traxler \& M. A. Gernsbacher (Eds.), Handbook of psycholinguistics (pp. 61-91). Oxford: Elsevier.

Ferreira, F., LaU, E. F., \& Bailey, K. G. D. (2004). Disfluencies, language comprehension, and tree adjoining grammars. Cognitive Science, 28, 721-749.

Finlayson, I. R., \& Corley, M. (2010). Disfluency in dialogue: An intentional signal from the speaker. Manuscript submitted for publication.

FORD, M. (1982). Sentence planning units: Implications for the speaker's representation of meaningful relations underlying sentences. In J. Bresnan (Ed.), The mental representation of grammatical relations (pp. 798-827). Cambridge, MA: MIT Press.

Fox Tree, J. E. (1995). The effects of false starts and repetitions on the processing of subsequent words in spontaneous speech. Journal of Memory \& Language, 34, 709-738.

Fox Tree, J. E. (2001). Listeners' uses of $u m$ and $u h$ in speech comprehension. Memory \& Cognition, 29, 320-326.

Fox Tree, J. E., \& Clark, H. H. (1997). Pronouncing "the" as "thee" to signal problems in speaking. Cognition, 62, 151-167.

Fromkin, V. A. (1971). The nonanomalous nature of anomalous utterances. Language, 47, 27-52.

Garrett, M. F. (1975). The analysis of sentence production. In G. Bower (Ed.), Psychology of learning and motivation (Vol. 9, pp. 133-177). New York: Academic Press.

Giedd, J. N., Blumenthal, J., Jefferies, N. O., Castellanos, F. X., Lui, H., ZiJdenbos, A., ET AL. (1999). Brain development during childhood and adolescence: A longitudinal MRI study. Nature Neuroscience, 2, 861-863.

Goldman-EIsLer, F. (1968). Psycholinguistics: Experiments in spontaneous speech. New York: Academic Press.

Gorfein, D. S., \& MacLeod, C. M. (2007). Inhibition in cognition. Washington, DC: American Psychological Association.

Hart, E. L., Lahey, B. B., Loeber, R., Applegate, B., \& Frick, P. J. (1995). Developmental change in attention-deficit hyperactivity disorder in boys: A four-year longitudinal study. Journal of Abnormal Child Psychology, 23, 729-749.

HASHER, L., \& ZACKS, R. T. (1988). Working memory, comprehension, and aging: A review and a new view. In G. H. Bower (Ed.), The psychology of learning and motivation (Vol. 22, pp. 193-225). New York: Academic Press.

HASHER, L., ZACKS, R. T., \& MAY, C. P. (1999). Inhibitory control, circadian arousal, and age. In D. Gopher \& A. Koriat (Eds.), Attention and performance XVII: Cognitive regulation of performance. Interaction of theory and application (pp. 653-675). Cambridge, MA: MIT Press.

James, L. E., Burke, D. M., Austin, A., \& Hulme, E. (1998). Production and perception of verbosity in young and older adults. Psychology \& Aging, 13, 355-367.

Kutas, M., \& Hillyard, S. A. (1980). Reading senseless sentences: Brain potentials reflect semantic incongruity. Science, 207, 203-205.

Lahey, B. B., Pelham, W. E., Loney, J., Lee, S. S., \& Willcutt, E. G. (2005). Instability of the DSM-IV subtypes of ADHD from preschool through elementary school. Archives of General Psychiatry, 62, 896902.

LaU, E. F., \& Ferreira, F. (2005). Lingering effects of disfluent material on comprehension of garden path sentences. Language \& Cognitive Processes, 20, 633-666.

Le Dorze, G., \& BedARD, C. (1998). Effects of age and education on the lexico-semantic content of connected speech in adults. Journal of Communication Disorders, 31, 53-71.

Levelt, W. J. M. (1983). Monitoring and self-repair in speech. Cognition, 14, 41-104.

LEVELT, W. J. M. (1989). Speaking: From intention to articulation. Cambridge, MA: MIT Press.

Lorch, E. P., Milich, R., Sanchez, R. P., van den Broek, P., Baer, S., HooKs, K., ET AL. (2000). Comprehension of televised stories in boys with attention deficit/hyperactivity disorder and nonreferred boys. Journal of Abnormal Psychology, 109, 321-330.

Martel, M. M., Nikolas, M., \& NigG, J. T. (2007). Executive functions in adolescents with ADHD. Journal of the American Academy of Child \& Adolescent Psychiatry, 46, 1434-1444. 
McCabe, D. P., Robertson, C. L., \& Smith, A. D. (2005). Age differences in Stroop interference in working memory. Journal of Clinical \& Experimental Neuropsychology, 27, 633-644.

Meyer, A. S., Wheeldon, L. R., \& Krott, A. (2007). Automaticity and control in language processing. New York: Psychology Press.

Mortensen, L., Meyer, A. S., \& Humphreys, G. W. (2006). Agerelated changes in object naming: A review. Language \& Cognitive Processes, 21, 238-290.

Nigg, J. T. (2001). Is ADHD a disinhibition disorder? Psychological Bulletin, 127, 571-598.

NIGG, J. T. (2006). What causes ADHD? Understanding what goes wrong and why. New York: Guilford.

O'Connell, D. C., \& Kowal, S. (2005). Uh and um revisited: Are they interjections for signaling delay? Journal of Psycholinguistic Research, 31, 555-576.

Oomen, C. C. E., \& Postma, A. (2001). Effects of time pressure on mechanisms of speech production and self-monitoring. Journal of Psycholinguistic Research, 30, 163-184.

Oviatt, S. (1995). Predicting spoken disfluencies during humancomputer interaction. Computer Speech \& Language, 9, 19-35.

Puig-Antich, J., \& Ryan, N. (1986). The schedule for affective disorders and schizophrenia for school-age children (Kiddie-SADS). Pittsburgh, PA: Western Psychiatric Institute and Clinic.

PURVIS, K., \& TANNOCK, R. (1997). Language abilities in children with attention deficit hyperactivity disorder, reading disabilities, and normal controls. Journal of Abnormal Child Psychology, 25, 133-144.

Rashid, F. L., Morris, M. K., \& Morris, R. (2001). Naming and verbal memory skills in adults with attention deficit hyperactivity disorder and reading disability. Journal of Clinical Psychology, 57, 829-838.

Redmond, S. M. (2004). Conversational profiles of children with ADHD, SLI, and typical development. Clinical Linguistics \& Phonetics, 18, 107-125.

Salthouse, T. A., \& Meinz, E. J. (1995). Aging, inhibition, working memory, and speed. Journals of Gerontology, 50B, 297-306.

SAndson, J., Obler, L. K., \& Albert, M. L. (1987). Language changes in healthy aging and dementia. In S. Rosenberg (Ed.), Advances in applied psycholinguistics (pp. 264-292). New York: Oxford University Press.

Sattler, J. M. (2001). Assessment of children: Cognitive applications (4th ed.). San Diego: Author.

Schachar, R., Tannock, R., Marriott, M., \& Logan, G. D. (1995). Deficient inhibitory control and attention deficit hyperactivity disorder. Journal of Abnormal Child Psychology, 23, 411-437.

Schmitter-Edgecombe, M., Vesneski, M., \& Jones, D. W. R. (2000). Aging and word-finding: A comparison of spontaneous and constrained naming tests. Archives of Clinical Neuropsychology, 15, 479-493.

Shewan, C. M., \& Henderson, V. L. (1988). Analysis of spontaneous language in the older normal population. Journal of Communication Disorders, 21, 139-154.
Shriberg, E. (1996). Disfluencies in Switchboard. In Proceedings of the International Conference on Spoken Language Processing (ICSLP) (Addendum, pp. 11-14). Philadelphia.

Smith, V., \& Clark, H. H. (1993). On the course of answering questions. Journal of Memory \& Language, 32, 25-38.

Studebaker, G. A. (1985). A "rationalized" arcsine transform. Journal of Speech \& Hearing Research, 28, 455-462.

WeCHSLER, D. (1997a). Wechsler Adult Intelligence Scale-Third Edition (WAIS-III). San Antonio, TX: Psychological Corporation.

WeCHSLER, D. (1997b). Wechsler Intelligence Scale for Children-Fourth Edition (WISC-IV). San Antonio, TX: Psychological Corporation.

WeChSLER, D. (2001). Wechsler Individual Achievement Test-Second Edition (WIAT-II). San Antonio, TX: Psychological Corporation.

Willcutt, E. G., Pennington, B. F., \& DeFries, J. C. (2000). Etiology of inattention and hyperactivity/impulsivity in a community sample of twins with learning difficulties. Journal of Abnormal Child Psychology, 28, 149-159.

\section{NOTES}

1. Christenfeld and Creager (1996) conducted a study on the rate of um production based on alcohol consumption. They found that increased alcohol consumption resulted in a decreased likelihood of producing $u m s$, an effect they attributed to people's being less self-conscious (i.e., less inhibited) after drinking. However, it could also be the case that more intoxicated people plan less and, therefore, produce fewer filled pause disfluencies.

2. Participants in the study also completed a working memory span task (Engelhardt, Nigg, Carr, \& Ferreira, 2008; McCabe, Robertson, \& Smith, 2005). The results, however, showed no significant correlations with disfluencies, and so we chose not to present these data in this article. We will provide the analyses and results to interested readers upon request to the first author.

3. The ambiguous verbs can be used in the active past tense (The man moved the chair), as an active past participle (The man had moved the chair), or in the passive voice (The chair was moved by the man). The participle verbs can be used as an active past participle (The girl had ridden the bike) or in the passive voice (The bike was ridden by the girl). The active past tense is not available with participle verbs (i.e., *The girl ridden the bike), and so these verbs have one less syntactic structure available, as compared with ambiguous verbs.

4. There were 93 utterances that were lost due to problems with the audio recording equipment and/or because the sentence was inaudible.

5. The high correlation between age and education is due to the compulsory education system up to 16 years of age. Our sample was also predominantly (i.e., 2:1) adolescents, which necessarily implies a high correlation between age and education.

(Manuscript received August 14, 2009; revision accepted for publication December 7, 2009.) 